\title{
Semantic Characterization of Context of Use and Contents for User-Centric Media Retrieval
}

\author{
Juan Carlos Yelmo García ${ }^{1}$, Yod Samuel Martín García ${ }^{1}$, \\ Beatriz San Miguel González ${ }^{1}$ \\ ${ }^{1}$ Universidad Politécnica de Madrid, Ciudad Universitaria s/n, 28040 Madrid, Spain \\ \{jcyelmo, samuelm, smiguel\} edit.upm.es
}

\begin{abstract}
When users access online media, they need and desire to get an experience tailored to their specific, personal context and situation. This is becoming more and more relevant with the ever-increasing amount of available contents users may choose from. In order to provide user-centric functionalities (such as relevant searches, content adaptation, customization and recommendation), both the annotation of contents with semantically rich metadata and an accurate model of the individual users and their respective contexts of use are needed. In this context, we propose a solution to automatically characterize both the context of use and the contents. It provides dynamic, adaptive user models, with explicit and implicit information; as well as content descriptors that may be later used to match the most suitable contents for each user. Users always keep a pivotal role throughout the whole process: providing new contents, contributing to moderated folksonomies, overseeing their own user model, etc.
\end{abstract}

Keywords: annotation, metadata, user model, content description, adaptation, customization, personalized search.

\section{Introduction: the need for semantic characterization}

Users are already accustomed to access contents anywhere, anytime and from any device. Nowadays, they expect to get an experience personalized to their specific preferences and needs -which may also vary with their context or the tasks they are performing at each moment. The answer to this demand lies in user-centric tools, which adapt their functionalities to each user, and keep them in permanent control of the process, whereas reducing the cognitive load to a minimum.

The contents of this paper belong to a broader project where a multimedia search engine is being developed [1]. We are presenting a work in progress that is aimed at offering a framework to enhance existing search functionalities with user-centric features, as well as to provide new operations, namely:

- User-centric search: defined as the selection of a reduced subset of contents from a large repository, based on some structured or unstructured indications provided by the user (in the form of a search query). Implicit inputs from the user model may improve search accuracy, relevance and correction. 
- Recommendation: same as search, but without any active intervention from the user (recommendation is push-mode, initiated by the platform without any other inputs).

- Customization: selection of one type of media from a reduced set of equivalent contents (e.g., versions of the same content)

- Adaptation: transformation of a concrete input content (this is the only operation where the content itself is delivered to the user, rather than mere pointers to it).

The foundations of the user-centric features are the same for each operation. The search engine relies on rich, semantic descriptions of both the user accessing it and the contents from a (distributed or centralized) repository. Given those descriptions, a match operation is performed that selects the best available contents for the particular user. Consequently, a structured knowledge needs to be obtainable about both the users (to know their needs and preferences) and the contents (to assess how they fit for a specific user) [2]. Specifically, we propose a semantic framework that captures, represents and stores those two kinds of knowledge, so that the engine may use it to provide user-centric functionalities. This framework is ancillary to the search engine, providing a repository of user and content metadata that may be applied to usercentric functionalities. Figure 1 summarizes all the elements of our framework, how they interrelate and connect to each other and the role the user plays with respect to each component.

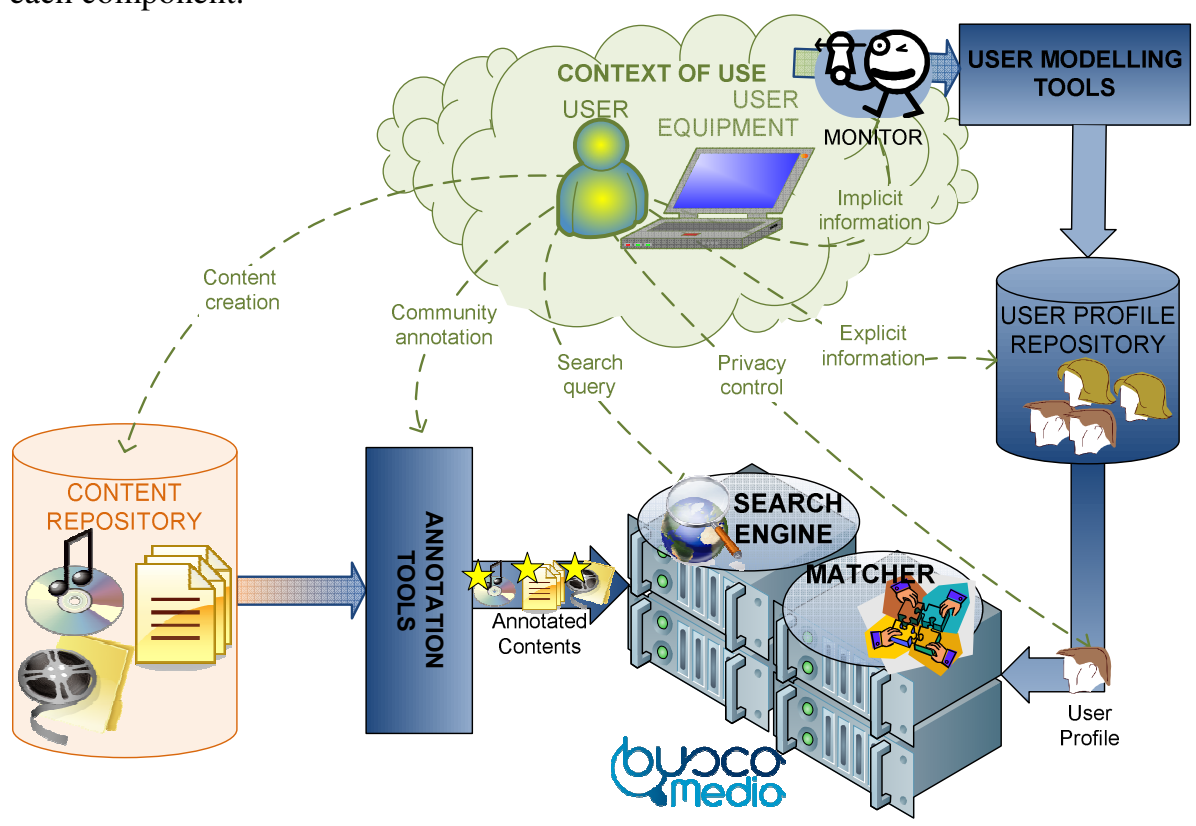

Fig. 1. A Search Engine is augmented with a Matcher module that provides user-centric features. The Matcher relies on information about both contents (provided by the annotation tools) and users (retrieved from the User Profile Repository). Users may play an active role in all the stages of this process. 


\section{Modelling the user and the context of use}

In order to provide the aforementioned user-centric functionalities, the search engine requires a model of each of the individual users accessing it [3]. This model is a computational description of a certain user in a system that includes relevant information of the user, providing a structured knowledge of their activities, preferences and capacities. To get a real picture, our user models not only deal with users themselves, but they also cover the rest of the elements appearing in their context of use: knowledge, expertise, goals, culture, preferences, behaviour, dexterous and sensory capabilities, organization, ambience, software and hardware platform, etc.

Next, we describe how this information is collected and we introduce the user model we are considering in our engine, together with the research challenges it might entail.

Capturing User Information. We may distinguish two types of user information regarding the way it is gathered:

- Explicit information: Users personally input it through forms or opinion polls. It may include demographic information, ratings, written opinions, etc.

- Implicit information: It is extracted from user activity or user interaction, without their intervention. Implicit information related to user preferences may be inferred from user browsing history or search logs (previously stored).

It might seem obvious that users themselves might generate more accurate and real information; however, they are not always willing to contribute and may even provide inaccurate information. This problem lies in the very essence of the explicit information: it may overload users who have to provide it. Users need good reasons to contribute -the fact being many web sites demonstrate these reasons can be actually found. We consider that the enhanced user experience and the benefits users can obtain from our user-centric functionalities may motivate them and ensure they keep their profiles accurate and updated. We aim to provide easy and simple interfaces that capture accurate information without overloading users or annoying them with too many questions; as well as offer incentives that motivate users to take an active part in the control of their own model. 
User model definition. We propose to merge explicit and implicit information to provide a dynamic user model controlled by the users themselves. We consider that users should be individually modelled as unique entities, since the differences among them are relevant enough in the scope of our search engine, where they require particular things. Dynamic user models are required because they do not keep the same information over time and they can be modified, amplified or improved to adapt to circumstances -and therefore, to the different users. In short, the major advantage of these models is just this last point: they allow characterizing users individually, since they adapt better to different users [4]. We may anticipate that several approaches we will employ will be based on the probabilistic inference of the user features (e.g. Bayesian networks, clustering around user stereotypes, collaborative filtering, etc).

Furthermore, as we mentioned before, we aim at providing users with control of their own user model. In this sense, based on the work of [5], we propose to incorporate appropriate interfaces that clearly present user information stored through an open user model. This open model also incorporates tools that allow users to oversee, modify and delete their information. It is important to point out that the user information that users can control is not only explicit information stored, but also information inferred by the engine. Furthermore, by changing this information, users may feed explicit information back to their user model, whose accuracy and validity will thus increase. If conflicts between explicit and implicit information appear, they will be solved probabilistically, nonetheless, the users will keep the last word on which information the model stores about them.

Users may benefit as well from this open model, since they can control and safeguard their privacy by watching, modifying and deleting their personal data in a transparent and clear way, which we think could be a reason to increase their participation in the collection of user information.

\section{Annotating the contents}

Three issues must be tackled regarding content annotations: their scope, their generation and their representation.

Scope: what should the metadata denote? In the scope of our project, we aim to get descriptors that: (a) are relevant to the operations mentioned in section 1, and (b) can entail constraints upon the context of use. For instance, we are considering these kinds of descriptors:

- Semantic labels: topics addressed by the content, domain of the context, named entities appearing in the content, etc.

- Context: date and location depicted in the content.

- Presentation characteristics: rendering modalities, media format, and natural language. 
Generation: how do we get metadata? In order to generate the content description, a direct option would be to analyze the contents every time they are being accessed; however, this turns out impractical because of its associated computational load. Instead, we propose a threefold approach to generate metadata used to annotate contents:

1. Manual or assisted annotation by content producers. Ideally, contents would be provided with semantic annotations attached during production (be by either the author or another member of the producer's team). However, this is not always feasible, especially when contents are created by non-professional prosumers [6].

2. Content augmentation by the community. Metadata may be adjoined to contents after they have been created. This may leverage on crowd wisdom to have annotations provided by users, each of whom may add a small bit of metadata. Examples of this practice are the folksonomies [7] (that label contents with semantic tags) and social accessibility [8] (where contents are enriched with hints for assistive technologies). We expect to have community annotations rely on automatic moderation tools to funnel the community, so as to ensure the quality and relevance of the annotations they generate and constrain them to partially controlled vocabularies that ease further processing.

3. Automated generation and storage of annotations by analytic tools. The cheapest way to add annotations is the automation of this process. The process may rely on external tools that perform activities ranging from the mere extraction of embedded metadata to linguistic analysis, object recognition, heuristic accessibility evaluation, etc.

Representation: how is metadata stored and transmitted? The metadata that annotates contents should be represented in a standard way that allows it to be reused by any tool, disregarding what original content it refers to. We have considered several, complementary alternatives:

- Generic, vocabulary-independent models such as the Protocol for Web Description Resources [9] (POWDER), which allows for easily integrating information on different domains (accessibility, adult-oriented content restrictions, mobile access, etc.)

- Multimedia content description standards such as MPEG-7.

- Bibliographic information models such as Dublin Core, Machine Reading Cataloging (MARC), Medatata Object Description Schema (MODS), or the Text Encoding Initiative.

- Multimedia news-oriented metadata formats, such as the Information Interchange Module and NewsML.

We must remark that all these different formats are easy to integrate in a generic framework by leveraging on generic models (such as the aforesaid POWDER), since all of them are controlled vocabularies which also define a representation as XML. 


\section{Conclusions and future work}

We have explained how user-centric content search, selection and recovery tools may take advantage of a proper characterization of contents and users to improve their results. A work in progress has been presented to explain a framework that collects, stores and represents metadata that may be used by those tools. This framework may be qualified as user-centric, because not only it eases a personalized access to contents, but it also puts the users in control of the whole annotation process, letting them decide how involved they would be:

- Content annotation may range from being specified during production time, to being fully automated, to even rely on other users socially augmenting it.

- User models may be built up exclusively from inferred information, but they also rely on user-provided information. Moreover, privacy-concerned users may always limit and specify the information stored about them at each moment.

As the work herein presented continues, the annotation framework implementation will be completed, possibly integrating external tools to analyze contents and user logs, and finally they will be integrated into the search, selection and recovery tools.

Acknowledgments. The works herein presented are being developed as part of the Buscamedia project of the CENIT-E programme, with reference number CEN20091026, partially funded by the CDTI (Centro para el Desarrollo Tecnológico e Industrial), supported by the Spanish Ministry of Science and Innovation. The authors would like to thank the knowledge and the contributions of the Buscamedia consortium partners (www.cenitbuscamedia.es). This part of the project has been specifically contracted to Universidad Politécnica de Madrid by GFI Informática.

\section{References}

1. Cenit Buscamedia. Project Page, http://www.cenitbuscamedia.es/

2. Moura, J., Chainho, P., and Damásio, C. V. Semantically Enabled Framework for User Centric Profile Description, Search and Match. In Service-Oriented Computing - ICSOC 2007 Workshops: ICSOC 2007, international Workshops, Vienna, Austria, September 17, 2007, Revised Selected Papers, E. Nitto and M. Ripeanu, Eds. Lecture Notes In Computer Science, vol. 4907, pp. 326-335, Springer-Verlag, Berlin, Heidelberg (2007).

3. Rich, E. 1999. Users are individuals: individualizing user models. Int. J. Hum.-Comput. Stud. vol.51, no. 2, pp. 323-338, Aug. 1999.

4. Gaudioso, E., Boticario, J. G., User Data Management and Usage Model Acquisition in an Adaptive Educational Collaborative Environment. In: Adaptive Hypermedia and Adaptive Web-Based Systems, LNCS, vol. 2347/2006, pp. 143-152, Springer Berlin / Heidelberg (2006).

5. Ahn, J., Brusilovsky, P., Grady, J., He, D., and Syn, S. Y. Open user profiles for adaptive news systems: help or harm?. In Proceedings of the 16th international Conference on World Wide Web (Banff, Alberta, Canada, May 08 - 12, 2007). WWW '07, pp. 11-20. ACM, New York, NY (2007).

6. Participative Web and User-Created Content: Web 2.0, Wikis and Social Networking. SourceOCDE Science et technologies de l'information, 2007, 15. OECD Organisation for Economic Co-operation and Development (2007). 
7. Halpin, H., Robu, V., and Shepherd, H. The complex dynamics of collaborative tagging. In Proceedings of the 16th international Conference on World Wide Web (Banff, Alberta, Canada, May 08 - 12, 2007). WWW '07, pp. 211-220. ACM, New York, NY (2007).

8. Sato, D., Kobayashi, M., Takagi, H., and Asakawa, C. 2010. Social accessibility: the challenge of improving web accessibility through collaboration. In Proceedings of the 2010 international Cross Disciplinary Conference on Web Accessibility (W4a) (Raleigh, North Carolina, April 26 - 27, 2010). W4A '10. ACM, New York, NY (2010).

9. Archer, P., Smith, K., Perego, A. (eds.), Protocol for Web Description Resources (POWDER): Description Resources, W3C Recommendation. W3C (2009). http://www.w3.org/TR/powder-dr/ 